\title{
ARBORIZAÇÃO URBANA DA CIDADE DE ITAPURANGA, GOIÁS
}

Rosilane Fernanda de Faria ${ }^{1}$, Valéria Rodrigues de Sousa², Sabrina do Couto de Miranda ${ }^{3}$

\section{RESUMO}

A arborização urbana deve respeitar valores culturais, ambientais e a memória da cidade. Contudo, a maioria das cidades negligencia a arborização favorecendo plantios irregulares com predomínio de espécies exóticas. Para se planejar a arborização primeiramente é preciso conhecer a composição florística local. Assim, este estudo objetivou analisar a composição florística da vegetação encontrada nas vias públicas de quatro bairros, com diferentes décadas de criação, da cidade de Itapuranga-Goiás. Foram sorteados bairros criados a partir da década de 1970. Em cada bairro foram amostradas cinco ruas onde todos os indivíduos lenhosos com diâmetro da base $\geq 5 \mathrm{~cm}$ tiveram seus diâmetro e altura mensurados. No total foram amostrados 738 indivíduos, 55 espécies e 24 famílias. Fabaceae apresentou o maior número de espécies (11). As espécies Syzygium malaccense (L.) Merr. \& L.M. Perry e Licania tomentosa (Benth.) Fritsch juntas representaram $66 \%$ do total de indivíduos plantados nas vias públicas, fator que influenciou a biodiversidade local. Quanto à similaridade, os bairros São Sebastião do Xixá (1970) e Parque Alvorada (1980) foram os mais similares entre si. A análise dos bairros mostrou heterogeneidade florística, variação devido à falta de planejamento da arborização.

Palavras-chave: Levantamento Florístico; Vias Públicas; Diversidade; Planejamento.

Recebido em 08.01.2013 e aceito em 27.03.2014

1 Graduada em Ciências Biológicas, Universidade Estadual de Goiás (UEG), Unidade Universitária de Itapuranga-GO. Itapuranga-Goiás. rosy.fernanda@gmail.com

2 Graduada em Ciências Biológicas, Universidade Estadual de Goiás (UEG), Unidade Universitária de Itapuranga-GO. Itapuranga-Goiás.valeria.bio.sousa@gmail.com

3 Professora de Botânica, Universidade Estadual de Goiás (UEG), Unidade Universitária de Itapuranga. Goiânia-Goiás. sabrina_miranda@yahoo.com.br 


\section{URBAN FORESTRY OF ITAPURANGA CITY, GOIÁS STATE}

\section{ABSTRACT}

The urban forestry should respect cultural, environmental and memory values of the city. However, most cities neglect this important component of environment management and the consequences are irregular plantations and the predominance of the exotic species in most of the cases. The first step to an effective afforestation plan is to know the local floristic composition. This study aimed to analyze the floristic composition of the vegetation found on public roads in four neighborhood, with different decades of creation, in the Itapuranga city, Goiás State. The neighborhood were created from the 1970s. In each neighborhood were sampled five streets where all woody plants with diameter of base $\geq 5 \mathrm{~cm}$ were sampled (diameter and height). In total were sampled 738 individuals, 55 species and 26 families. Fabaceae was the family with the highest number of species (11). The species Syzygium malaccense (L.) Merr. \& L.M. Perry and Licania tomentosa (Benth.) Fritsch together represented $66 \%$ of total individuals, a factor that influenced the local biodiversity. As for similarities, the neighborhood São Sebastião Xixá (1970) and Parque Alvorada (1980) were the most similar. The analysis showed floristic heterogeneity in the neighborhood and that variation due to lack of planning afforestation.

Keywords: Floristic Survey; Public Roads; Diversity; Planning.

\section{INTRODUÇÃO}

As árvores em áreas urbanas geram inúmeros benefícios que, indiscutivelmente, influenciam o bem estar do homem (BATISTEL et al., 2009), porém se instaladas em locais inadequados podem também gerar prejuízos e representarem focos de conflitos nas cidades (SILVA FILHO et al., 2002; COLETTO et al., 2008; MAZZAROTTO, et al., 2011). O planejamento da arborização é indispensável para o desenvolvimento urbano (DANTAS; SOUZA, 2004). Contudo, a maioria das cidades brasileiras negligencia a arborização urbana (BATISTEL et al., 2009), o que favorece plantios irregulares de espécies incompatíveis com determinado local (SILVA FILHO; BORTOLETO, 2005), bem como o predomínio de espécies exóticas. 
A arborização urbana bem planejada deve respeitar valores culturais, ambientais e a memória da cidade (SÃO PAULO, 2005). Além disso, o planejamento da arborização visa aperfeiçoar os múltiplos benefícios que as florestas urbanas proporcionam ao meio, tais como: disponibilidade de sombra, ameniza a poluição sonora, melhora a qualidade do ar, diminui a ação dos ventos, fornece abrigo e alimento à fauna, fixação de carbono, dentre outros (RODIGUES et al., 2002; BATISTEL et al., 2009; MUNEROLI; MASCARÓ, 2010).

Para se planejar a arborização primeiramente é preciso conhecer a composição florística local. Os resultados deste levantamento subsidiarão tanto o planejamento quanto o manejo da arborização (SILVA et al., 2007). Outra etapa importante é a escolha das espécies a serem plantadas. Deve-se decidir com base em considerações técnicas qual espécie deve ser plantada em determinado local (CEMIG, 2011). Uma escolha bem feita diminui os custos com manutenção (PAIVA, 2000) e exalta os benefícios da arborização.

Como dito anteriormente, a arborização urbana deve respeitar o contexto ambiental da cidade. Nesse sentido, deve-se priorizar o plantio de espécies nativas que valorizem a identidade do bioma no qual a cidade está inserida. A utilização de espécies nativas contribui ainda para o aumento nas taxas de sobrevivência após o plantio, maior longevidade das árvores no meio urbano, redução com custos de manutenção e contato da população local com a vegetação nativa (COSTA E LIMA; SILVA JÚNIOR, 2010).

Neste contexto, este trabalho objetivou descrever a composição florística da vegetação lenhosa urbana encontrada nas vias públicas de quatro bairros da cidade de Itapuranga-Goiás. Foram avaliados bairros criados em diferentes décadas com o intuito de verificar possíveis mudanças na arborização ao longo do tempo.

\section{MATERIAIS E MÉTODOS}

Este estudo foi realizado na cidade de Itapuranga-GO (15³3'18,8'S e 4956'10,4"WO) (Figura 1) que dista 153 km da capital do estado (Goiânia) e se localiza na região do Vale do São Patrício, Centro-Oeste do estado de Goiás. De acordo com Instituto Brasileiro de Geografia e Estatística (IBGE, 2010) a área territorial do município é de $1.276,48 \mathrm{~km}^{2}$, com altitude média de $651 \mathrm{~m}$ e população estimada em 26.125 habitantes. A temperatura média para o período de 2002 a 2012 variou de $20,6^{\circ} \mathrm{C}$ a $33,2^{\circ} \mathrm{C}$, com umidade relativa do ar média de 63,8\% (INMET, 2012). 
O município foi criado em 1933 recebendo o nome de Xixá em virtude da celebração da primeira missa campal à sombra de um enorme Xixazeiro. Passando a ser denominado Itapuranga somente em 1953 (IBGE, 2010).

Figura 1. Localização geográfica da cidade de Itapuranga, Goiás. Fonte: IBGE Figure 1. Geographical location of the Itapuranga city, Goias State
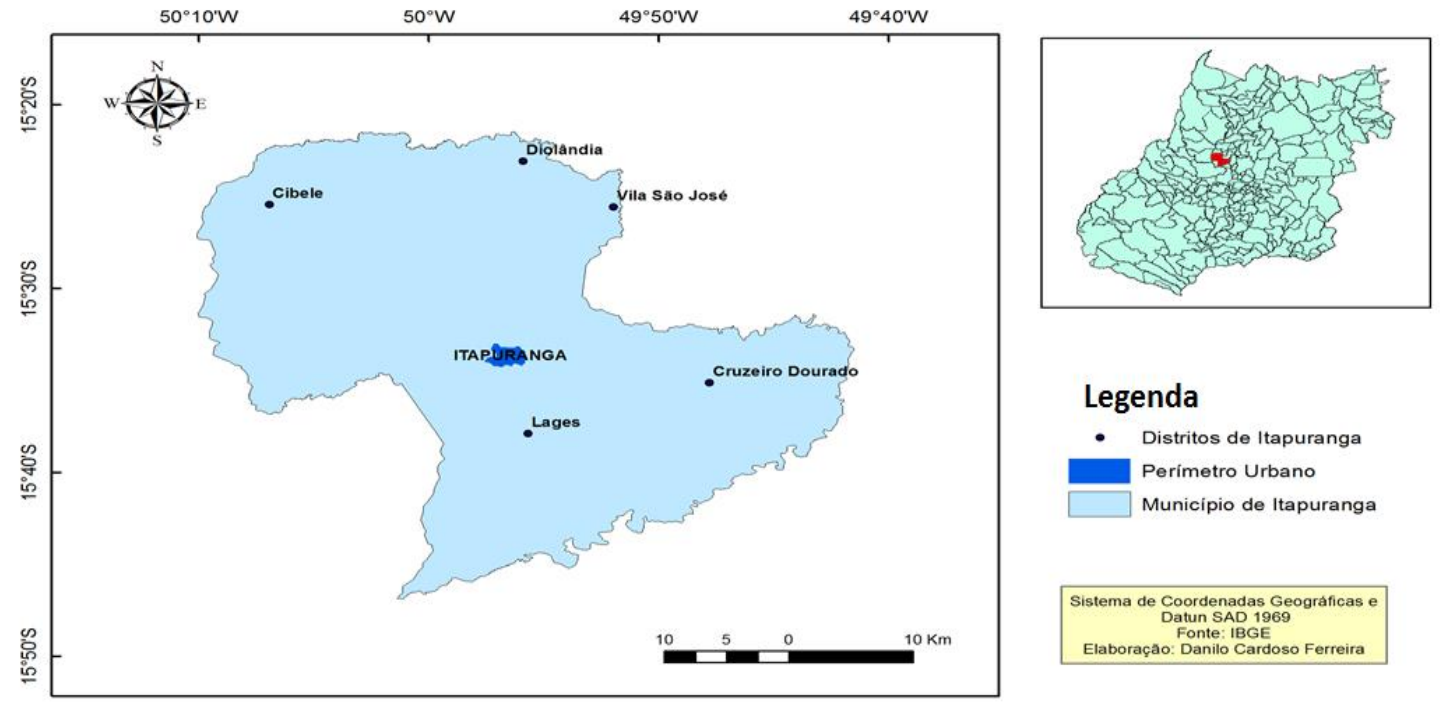

\section{Legenda}

- Distritos de Itapuranga Perímetro Urbano Municipio de Itapuranga

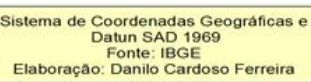

A cidade de Itapuranga está inserida no domínio do Cerrado, segundo maior bioma em extensão geográfica (FELFILI; SILVA JÚNIOR, 2005). O Cerrado é caracterizado por mosaico heterogêneo de fitofisionomias que variam desde formas campestres, savânicas até florestais (RIBEIRO; WALTER, 2008). No entorno do município predominavam fitofisionomias florestais de Cerrado, tais como cerradão e florestas estacionais (matas secas). Atualmente, estas formações vegetacionais são manchas pequenas e isoladas circundadas, principalmente, por plantações de cana de açúcar e eucaliptos.

Dados sobre o período de criação de cada setor foram obtidos em registros no cartório da cidade. Posteriormente, foram sorteados quatro bairros na sequência histórica de sua implantação, considerando as décadas de 1970, 1980, 1990 e 2000 (Tabela 1). Em cada bairro selecionou-se uma rua e, a partir desta, outras quatro foram amostradas (Tabela 1). Por se tratar de um estudo florístico, e não fitossociológico, não computamos a extensão em metros da amostragem. Ao longo das calçadas das ruas selecionadas amostrou-se todos os indivíduos lenhosos, exceto palmeiras, com diâmetro da base (DB), medido a 30 $\mathrm{cm}$ do solo, $\geq 5 \mathrm{~cm}$. Os indivíduos amostrados tiveram seus diâmetros e alturas mensurados, respectivamente, com suta de alumínio e vara graduada em metros. A altura total foi considerada da base do tronco até o ramo mais alto ou folhagem. 
Tabela 1. Bairros e ruas selecionados para amostragem dos indivíduos lenhosos nas vias públicas da cidade de Itapuranga-GO

Table 1. Sectors and streets selected for woody plants sampling on public roads in Itapuranga city, Goias State

\begin{tabular}{|c|c|c|}
\hline Bairros & Data de criação & Ruas \\
\hline São Sebastião do Xixá & $17 / 07 / 1970$ & $\begin{array}{l}\text { Rua } 49 \\
\text { Rua 36 } \\
\text { Rua 33 } \\
\text { Rua 26 } \\
\text { Rua 47 }\end{array}$ \\
\hline Parque Alvorada & 24/02/1983 & $\begin{array}{c}\text { Rua 63 } \\
\text { Rua 08 } \\
\text { Rua 09 } \\
\text { Rua A } \\
\text { Rua 07 }\end{array}$ \\
\hline Setor Comercial & 07/11/1991 & $\begin{array}{l}\text { Rua 05 } \\
\text { Rua 03 } \\
\text { Rua 04 } \\
\text { Rua 06 } \\
\text { Rua 02 }\end{array}$ \\
\hline Jardim Conde dos Arcos & $01 / 02 / 2000$ & $\begin{array}{c}\text { Rua José Araújo } \\
\text { Parreira } \\
\text { Rua Albatroz } \\
\text { Rua Xixá } \\
\text { Rua Alves de Faria } \\
\text { Rua Tocantins } \\
\end{array}$ \\
\hline
\end{tabular}

Em campo foram utilizadas fichas para anotações dos dados coletados com as seguintes informações: data da coleta, nome do bairro, nome da rua, sentido da coleta, nome das espécies e características fenológicas. Os indivíduos foram identificados por meio de consultas a bibliografias especializadas (LORENZI, 2008; MATOS; QUEIROZ, 2009; SILVA JÚNIOR; COSTA E LIMA, 2010) e visitas ao Herbário da Universidade de Brasília (UB). As coletas de dados se iniciaram em janeiro de 2012 e se encerraram em abril deste mesmo ano.

As espécies foram classificadas em famílias de acordo com o sistema Angiosperm Phylogeny Group III (APG III, 2009). Os nomes científicos das espécies e das famílias botânicas foram conferidos no site do "W3 Tropicos" (http://www.tropicos.org/). A origem das espécies amostradas e os domínios fitogeográficos de ocorrência foram conferidos nos sites "Lista de espécies da Flora do Brasil 2012" (http://floradobrasil.jbrj.gov.br/2012/) e "W3 Tropicos".

O índice de diversidade de Shannon e Wiener e uniformidade de Pielou (MAGURRAN; MCGILL, 2011) foram calculados com o auxílio do programa MVSP (KOVACH, 1993). A similaridade florística entre os bairros foi avaliada com base no índice de Sørensen, baseado na presença de espécies (MAGURRAN; MCGILL, 2011) e calculado 
pelo programa MVSP (KOVACH, 1993). Nunes et al. (2002) calcularam em 0,5 a mediana do índice de similaridade de Sørensen nas comparações entre 100 parcelas de cerrado no Distrito Federal, assim foram considerados elevados os valores acima de 0,5. A análise da distribuição de diâmetros foi realizada utilizando-se intervalo entre classes de $5,0 \mathrm{~cm}$ e para a altura intervalos de 1,0 m.

\section{RESULTADOS E DISCUSSÃO}

Nos quatro bairros estudados foram amostrados 738 indivíduos lenhosos distribuídos em 55 espécies, 51 gêneros e 24 famílias (Tabelas 2 e 3). Com base nestes dados podemos destacar a alta diversidade em nível genérico, pois do total de gêneros amostrados, 49 foram representados por apenas uma espécie. Somente os gêneros Annona, Citrus, Spondias e Syzygium foram representados por duas espécies cada (Tabela 2). Com relação às famílias, Fabaceae apresentou o maior número de espécies (11), seguida por Anacardiaceae com cinco espécies (Tabela 2). De acordo com Costa e Lima e Silva Júnior (2010), Fabaceae e Anacardiaceae foram famílias que se destacaram em número de espécies na arborização urbana implantada na década de 1960 no Plano Piloto em Brasília-DF. A alta representatividade de Fabaceae na arborização urbana deve-se à floração vistosa de suas espécies (COSTA; LIMA, 2009). No caso de Anacardiaceae várias espécies produzem frutos e pseudofrutos que são bastante apreciados pela população (SOUZA; LORENZI, 2008).

O levantamento florístico revelou que a arborização urbana de Itapuranga apresenta alta representatividade de apenas duas espécies, Syzygium malaccense que é exótica e Licania tomentosa nativa do Brasil, que representaram $66 \%$ do total de indivíduos levantados (Tabela 3). Esta última espécie consta na lista de espécies recomendadas e mais utilizadas na arborização de ruas e avenidas do Brasil (GUIA, 1988; ÁRVORES, 1999; SANTOS; TEIXEIRA, 2001; PIVETTA; SILVA FILHO, 2002). 
Tabela 2. Composição florística das vias públicas amostradas na cidade de Itapuranga-GO Table 2. Floristic composition of public roads sampled in the Itapuranga city, Goias State

\begin{tabular}{|c|c|c|}
\hline Famílias & Espécies & $\begin{array}{c}\text { Distribuição original } \\
\text { ou nativa }\end{array}$ \\
\hline \multirow{5}{*}{ Anacardiaceae } & Anacardium occidentale L. & $\mathrm{Ce}, \mathrm{Ca}, \mathrm{MA}, \mathrm{Am}, \mathrm{Pt}$ \\
\hline & Mangifera indica L. & $\mathrm{E}$ \\
\hline & Schinus molle L. & $\mathrm{Ce}, \mathrm{Pa}$ \\
\hline & Spondias mombin L. & $\mathrm{Am}, \mathrm{Ca}, \mathrm{Ce}, \mathrm{MA}$ \\
\hline & Spondias purpurea $\mathrm{L}$. & $\mathrm{Ca}$ \\
\hline \multirow{3}{*}{ Annonaceae } & Annona muricata L. & $E$ \\
\hline & Annona sp. & - \\
\hline & Rollinia mucosa (Jacq.) Baill. & Am, MA \\
\hline \multirow{2}{*}{ Apocynaceae } & Plumeria rubra L. & $\mathrm{E}$ \\
\hline & Thevetia neriifolia Juss. ex Steud. & $\mathrm{E}$ \\
\hline \multirow{3}{*}{ Bignoniaceae } & Spathodea campanulata P. Beauv. & $\mathrm{E}$ \\
\hline & Tabebuia rosea (Bertol.) A. DC. & $\mathrm{Ce}, \mathrm{MA}$ \\
\hline & Tecoma stans (L.) Juss. ex Kunth & $E$ \\
\hline Chrysobalanaceae & Licania tomentosa (Benth.) Fritsch & $\mathrm{Ca}$ \\
\hline \multirow{2}{*}{ Clusiaceae } & Clusia sp. & - \\
\hline & Garcinia gardneriana (Planch. \& Triana) Zappi & $\mathrm{Am}, \mathrm{Ca}, \mathrm{Ce}, \mathrm{MA}$ \\
\hline Combretaceae & Terminalia catappa L. & $\mathrm{E}$ \\
\hline Euphorbiaceae & Jatropha curcas L. & Am, Ca, MA \\
\hline \multirow{11}{*}{ Fabaceae } & Adenanthera pavonina $\mathrm{L}$. & $E$ \\
\hline & Anadenanthera peregrina (L.) Speg. & $\mathrm{Am}, \mathrm{Ca}, \mathrm{Ce}, \mathrm{MA}$ \\
\hline & Bauhinia variegata $\mathrm{L}$. & $E$ \\
\hline & Caesalpinia pluviosa DC. & MA \\
\hline & Cassia fistula L. & $E$ \\
\hline & Delonix regia (Bojer ex Hook.) Raf. & $E$ \\
\hline & Inga edulis var. parviflora Benth. & $\mathrm{Am}, \mathrm{Ca}, \mathrm{Ce}, \mathrm{MA}$ \\
\hline & Leucaena leucocephala (Lam.) de Wit & E \\
\hline & Ormosia sp. & - \\
\hline & Peltophorum dubium (Spreng.) Taub. & $\mathrm{Ce}$ \\
\hline & Pterogyne nitens Tul. & $\mathrm{Ca}, \mathrm{Ce}, \mathrm{MA}, \mathrm{Pa}$ \\
\hline Lamiaceae & Vitex polygama Cham & $\mathrm{Ca}, \mathrm{Ce}, \mathrm{MA}$ \\
\hline \multirow{3}{*}{ Lauraceae } & Cinnamomum sp. & - \\
\hline & Nectandra megapotamica (Spreng.) Mez & $\mathrm{Ce}, \mathrm{MA}$ \\
\hline & Persea americana Mill. & E \\
\hline Leguminosae & Erythrina sp. & - \\
\hline \multirow{2}{*}{ Malpighiaceae } & Lophanthera lactescens Ducke & $\mathrm{Am}$ \\
\hline & Malpighia glabra L. & E \\
\hline Malvaceae & Pachira aquatica Aubl. & $\mathrm{Am}$ \\
\hline
\end{tabular}




\begin{tabular}{|c|c|c|}
\hline Malvaceae & $\begin{array}{c}\text { Sterculia chicha A. St.-Hil. ex Turpin } \\
\text { Theobroma cacao L. }\end{array}$ & $\begin{array}{c}\mathrm{Ce} \\
\mathrm{Am}, \mathrm{MA}\end{array}$ \\
\hline Melastomataceae & Tibouchina granulosa (Desr.) Cogn & MA \\
\hline Meliaceae & Melia azedarach L. & $\mathrm{E}$ \\
\hline \multirow{3}{*}{ Moraceae } & Artocarpus heterophyllus Lam. & E \\
\hline & Ficus elastica Roxb. ex Hornem. & E \\
\hline & Morus nigra L. & E \\
\hline \multirow{3}{*}{ Myrtaceae } & Psidium guajava L. & $\mathrm{Am}, \mathrm{Ca}, \mathrm{Ce}, \mathrm{MA}$ \\
\hline & Syzygium cumini (L.) Skeels & E \\
\hline & Syzygium malaccense (L.) Merr. \& L.M. Perry & E \\
\hline Nyctaginaceae & Bougainvillea glabra Choisy & $\mathrm{Ca}, \mathrm{MA}$ \\
\hline Proteaceae & Grevillea banksii R. Br. & $\mathrm{E}$ \\
\hline \multirow{2}{*}{ Rubiaceae } & Genipa americana L. & $\mathrm{Am}, \mathrm{MA}, \mathrm{Ce}$ \\
\hline & Morinda citrifolia L. & $\mathrm{Am}, \mathrm{Ca}, \mathrm{Ce}, \mathrm{MA}, \mathrm{Pa}$ \\
\hline \multirow{2}{*}{ Rutaceae } & Citrus aurantium L. & E \\
\hline & Citrus limon (L.) Osbeck & E \\
\hline Sapotaceae & Pouteria torta (Mart.) Radlk. & $\mathrm{Am}, \mathrm{Ca}, \mathrm{Ce}, \mathrm{MA}$ \\
\hline Verbenaceae & Duranta repens L. & $\mathrm{Am}, \mathrm{Ca}, \mathrm{Ce}, \mathrm{MA}$ \\
\hline
\end{tabular}

Am-Floresta Amazônica, Ce-Cerrado, Ca-Caatinga, MA-Floresta Atlântica, Pa-Pampa, Pt-Pantanal, E-Exótica, DD-Domínio desconhecido e (-) Não encontrado.

A alta representatividade de apenas duas espécies na arborização reflete na biodiversidade urbana local, de acordo com a literatura recomenda-se não utilizar mais de $30 \%$ de uma única família de árvore, $20 \%$ de um único gênero e 10\% de uma única espécie na arborização (CEMIG, 2011). No entanto, o que ocorre na maioria das cidades é a predominância de poucas espécies na arborização urbana (PIVETTA; SILVA FILHO, 2002; BATISTEL et al., 2009; SERPA et al., 2009; SOUZA et al. 2011), dentre os fatores que explicam tal predominância podemos citar a facilidade na aquisição de sementes e produção de mudas, germinação sem restrições, crescimento rápido, dentre outros. 
Tabela 3. Parâmetros estruturais dos indivíduos lenhosos amostrados nas vias públicas da cidade de Itapuranga-GO. Onde: $\mathrm{DB}=$ valor médio do diâmetro da base, $\mathrm{H}=$ =valor médio da altura, $C V=$ coeficiente de variação

Table 3. Structural parameters of woody plants sampled on public roads in Itapuranga city, Goiás State. Where: $\mathrm{DB}=$ mean value of base diameter, $\mathrm{H}=$ mean value of height, $\mathrm{CV}=$ coefficient of variation

\begin{tabular}{|c|c|c|c|c|c|}
\hline Espécies & $\begin{array}{c}\text { № de } \\
\text { indivíduos }\end{array}$ & $\begin{array}{l}\text { DB } \\
(\mathbf{c m})\end{array}$ & $\begin{array}{c}\text { DB-CV } \\
(\%)\end{array}$ & $\begin{array}{l}\mathrm{H} \\
(\mathrm{m})\end{array}$ & $\begin{array}{c}\mathrm{H}-\mathrm{CV} \\
(\%)\end{array}$ \\
\hline Adenanthera pavonina L. & 1 & 24,83 & - & 6,40 & - \\
\hline Anacardium occidentale L. & 10 & 19,16 & 70,74 & 4,94 & 22,36 \\
\hline Anadenanthera peregrina (L.) Speg. & 1 & 35,65 & - & 12,00 & - \\
\hline Annona muricata L. & 5 & 21,39 & 43,63 & 5,16 & 22,38 \\
\hline Annona sp & 3 & 18,25 & 9,61 & 5,17 & 2,96 \\
\hline Artocarpus heterophyllus Lam. & 1 & 25,78 & - & 5,80 & - \\
\hline Bauhinia variegata $\mathrm{L}$. & 13 & 34,23 & 31,86 & 6,25 & 33,89 \\
\hline Bougainvillea glabra Choisy & 2 & 18,78 & 31,16 & 5,15 & 17,85 \\
\hline Caesalpinia pluviosa DC. & 1 & 56,02 & - & 6,30 & - \\
\hline Cassia fistula $\mathrm{L}$. & 2 & 38,99 & 21,36 & 9,00 & 18,86 \\
\hline Cinnamomum sp & 3 & 14,85 & 37,26 & 3,97 & 20,53 \\
\hline Citrus aurantium L. & 1 & 20,05 & - & 6,20 & - \\
\hline Citrus limon (L.) Osbeck & 4 & 11,54 & 41,80 & 3,83 & 25,77 \\
\hline Clusia sp & 1 & 9,55 & - & 2,90 & - \\
\hline Delonix regia (Bojer ex Hook.) Raf. & 3 & 50,93 & 49,15 & 10,15 & 46,83 \\
\hline Duranta repens L. & 6 & 19,26 & 45,87 & 4,00 & 18,03 \\
\hline Erythrina sp & 1 & 24,19 & - & 5,30 & - \\
\hline Ficus elastica Roxb. ex Hornem. & 8 & 36,61 & 60,92 & 5,86 & 29,77 \\
\hline $\begin{array}{l}\text { Garcinia gardneriana (Planch. \& Triana) } \\
\text { Zappi }\end{array}$ & 1 & 11,14 & - & 3,50 & - \\
\hline Genipa americana L. & 1 & 47,75 & - & 6,20 & - \\
\hline Grevillea banksii R. Br. & 2 & 19,89 & 3,39 & 4,80 & 23,57 \\
\hline Inga edulis var. parviflora Benth. & 2 & 33,10 & 4,08 & 5,05 & 7,00 \\
\hline Jatropha curcas L. & 1 & 16,87 & - & 3,00 & - \\
\hline Leucaena leucocephala (Lam.) de Wit & 1 & 16,87 & - & 6,40 & - \\
\hline Licania tomentosa (Benth.) Fritsch & 157 & 15,76 & 46,53 & 4,40 & 26,27 \\
\hline Lophanthera lactescens Ducke & 6 & 16,29 & 19,40 & 6,02 & 8,50 \\
\hline Malpighia glabra L. & 3 & 16,34 & 31,67 & 3,63 & 9,67 \\
\hline Mangifera indica L. & 26 & 31,15 & 58,86 & 6,31 & 33,64 \\
\hline Melia azedarach L. & 2 & 57,30 & 23,57 & 11,25 & 9,43 \\
\hline Morinda citrifolia L. & 1 & 6,37 & - & 2,70 & - \\
\hline Morus nigra L. & 1 & 31,51 & - & 5,90 & - \\
\hline Nectandra megapotamica (Spreng.) Mez & 7 & 21,19 & 24,53 & 5,93 & 11,18 \\
\hline Ormosia sp & 1 & 11,78 & - & 4,65 & - \\
\hline Pachira aquatica Aubl. & 31 & 44,29 & 38,11 & 6,21 & 32,59 \\
\hline Peltophorum dubium (Spreng.) Taub. & 1 & 35,97 & - & 6,30 & - \\
\hline Persea americana Mill. & 5 & 21,84 & 67,60 & 5,70 & 39,95 \\
\hline Plumeria rubra L. & 2 & 10,98 & 10,25 & 3,73 & 40,57 \\
\hline Pouteria torta (Mart.) Radlk. & 1 & 9,23 & - & 2,90 & - \\
\hline
\end{tabular}


Psidium guajava L.

Pterogyne nitens Tul.

Rollinia mucosa (Jacq.) Baill.

Schinus molle $\mathrm{L}$.

Spathodea campanulata P. Beauv.

Spondias mombin L.

Spondias purpurea $\mathrm{L}$.

Sterculia chicha A. St.-Hil. ex Turpin

Syzygium cumini (L.) Skeels

Syzygium malaccense (L.) Merr. \& L.M. Perry

Tabebuia rosea (Bertol.) A. DC.

Tecoma stans (L.) Juss. ex Kunth

Terminalia catappa L.

Theobroma cacao L.

Thevetia neriifolia Juss. ex Steud.

Tibouchina granulosa (Desr.) Cogn

Vitex polygama Cham

Total

\begin{tabular}{ccccc}
6 & 20,42 & 24,83 & 4,85 & 7,80 \\
1 & 17,19 & - & 5,50 & - \\
2 & 14,80 & 74,51 & 4,08 & 70,28 \\
6 & 26,21 & 28,84 & 5,25 & 28,73 \\
5 & 45,33 & 37,90 & 8,50 & 23,97 \\
1 & 40,74 & - & 9,50 & - \\
1 & 37,24 & - & 6,00 & - \\
1 & 13,37 & - & 6,00 & - \\
22 & 36,03 & 28,82 & 6,44 & 27,22 \\
333 & 18,64 & 43,62 & 5,36 & 27,50 \\
5 & 24,26 & 13,23 & 6,10 & 20,04 \\
1 & 13,37 & - & 3,00 & - \\
27 & 29,32 & 33,59 & 5,87 & 22,63 \\
1 & 13,05 & - & 3,70 & - \\
4 & 13,69 & 24,24 & 3,81 & 24,83 \\
2 & 10,98 & 18,45 & 3,95 & 5,37 \\
2 & 17,83 & 70,71 & 5,30 & 58,70 \\
\hline 738 & 21,61 & 57,95 & 5,32 & 31,99 \\
\hline
\end{tabular}

Outro fator que influenciou na biodiversidade local foi o grande número de espécies representadas por apenas um indivíduo e, portanto, raras na arborização urbana da cidade de Itapuranga. Do total de espécies levantadas, 22 (40\% do total) foram amostradas com um único indivíduo (Tabela 3). Dentre estas, destacamos Sterculia chicha, cujo nome popular (Xixá) foi dado à cidade na época de sua criação, e Pouteria torta, espécie comum em áreas de cerrado sentido restrito e cerradão (SILVA JÚNIOR, 2012). São espécies nativas do Cerrado, portanto, sugere-se que estas espécies sejam priorizadas nos plantios, levando-se em consideração locais adequados para os mesmos resgatando assim a identidade histórica e ambiental da cidade.

Quanto à origem das espécies, a maioria (54\%) é nativa do Brasil, fator positivo da arborização local. Verificou-se que dentre as nativas 34\% pode ser encontrada no Cerrado, bioma no qual o município de Itapuranga está inserido (Tabela 2). A utilização de espécies nativas auxilia na preservação da biodiversidade local (ISERNHAGEN et al., 2009; CEMIG, 2011). Itapuranga conta com viveiro municipal que produz cerca de 25.000 mudas/ano e utiliza cerca de 60 espécies, entre nativas e exóticas. Segundo comunicação pessoal com o responsável pelo viveiro, não há planejamento quanto à coleta e beneficiamento das sementes. Sementes e frutos são trazidos para plantio pelo Secretário de Meio Ambiente Municipal e, posteriormente, as mudas são plantadas ou distribuídas aleatoriamente para os habitantes da cidade. Assim, sugere-se que a prefeitura, em parceria com a universidade, produza mudas de espécies nativas da região, com certificação, que poderão ser utilizadas em novos plantios. 
Com base nos dados estruturais dos indivíduos lenhosos amostrados, verificou-se que a média dos diâmetros foi $21,6 \mathrm{~cm}$ com coeficiente de variação (CV) de $58 \%$ (Tabela 3). Já a altura variou menos, média de $5,3 \mathrm{~m}$ e 32\% de CV (Tabela 3). Os resultados obtidos mostram que a arborização urbana de Itapuranga é constituída principalmente por árvores de pequeno porte (SÃO PAULO, 2005) ou indivíduos jovens.

A distribuição dos diâmetros mostra que os indivíduos lenhosos estão representados em todas as classes diamétricas (Figura 2A). É possível encontrar tanto árvores com baixos diâmetros, provavelmente indivíduos jovens, quanto árvores com diâmetros acima de $30 \mathrm{~cm}$, indivíduos bem estabelecidos. A análise da distribuição de altura mostrou que arborização urbana de Itapuranga é caracterizada principalmente por indivíduos lenhosos com altura entre 4,2 e 6,1 m (Figura 2B), estas duas classes abrigaram 58\% dos indivíduos, o que ratifica que a arborização é composta principalmente por indivíduos de menor porte (SÃO PAULO, 2005). Este resultado deve-se à presença de fiação elétrica em ambos os lados das vias públicas o que impede ou dificulta o plantio de árvores de maior porte nestes locais.

A análise florística dos bairros mostrou que os mais antigos, criados nas décadas de 1970 e 1980, apresentaram maior número de indivíduos arbóreos plantados nas vias públicas, acima de 220 (Tabela 4) e maior riqueza (Tabela 4). No entanto, foram também os bairros que apresentaram maior percentual de espécies exóticas, 66\% no São Sebastião do Xixá e 69\% no Parque Alvorada.

O bairro Jardim Conde dos Arcos, criado na década de 2000, foi o que apresentou menor número de indivíduos plantados (Tabela 4), fato relacionado ao período de criação. Vários indivíduos não foram amostrados, pois não apresentaram o diâmetro mínimo de inclusão. Fato interessante é que este bairro foi o único que apresentou maior percentual de nativas plantadas (53\%) em comparação às exóticas e maior índice de diversidade $\left(H^{\prime}=2,19\right)$, a diversidade alfa é importante no planejamento, pois diminui os riscos de perdas por motivos de disseminação de pragas e doenças (COSTA; LIMA, 2009; CEMIG, 2011). Com base nos resultados apresentados sugere-se que a arborização dos setores menos diversificados (Parque Alvorada e Setor Comercial) seja manejada evitando-se assim problemas futuros relacionados à arborização urbana. 
Figura 2. Distribuição em classes de diâmetro (A) e de altura (B) dos indivíduos lenhosos amostrados nas vias públicas da cidade de Itapuranga-GO

Figure 2. Distribution in diameter class (A) and height (B) of woody plants sampled on public roads in Itapuranga city, Goias State

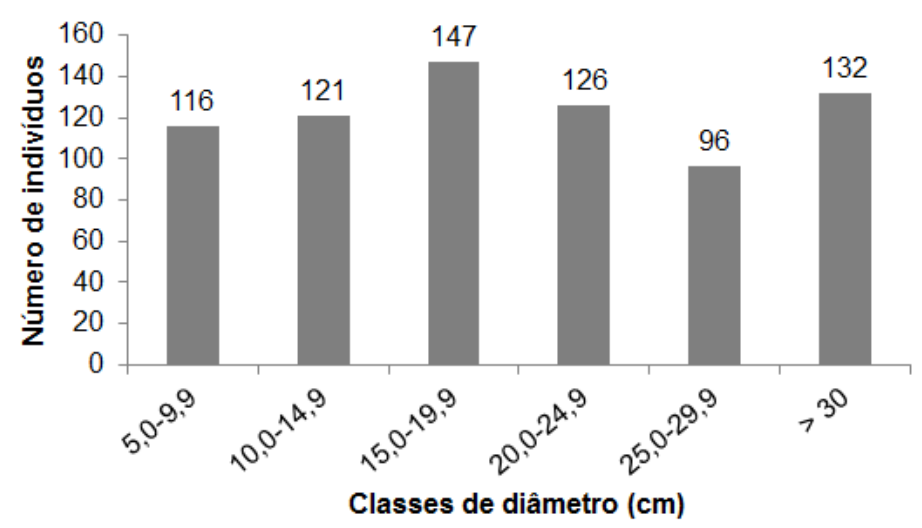

A

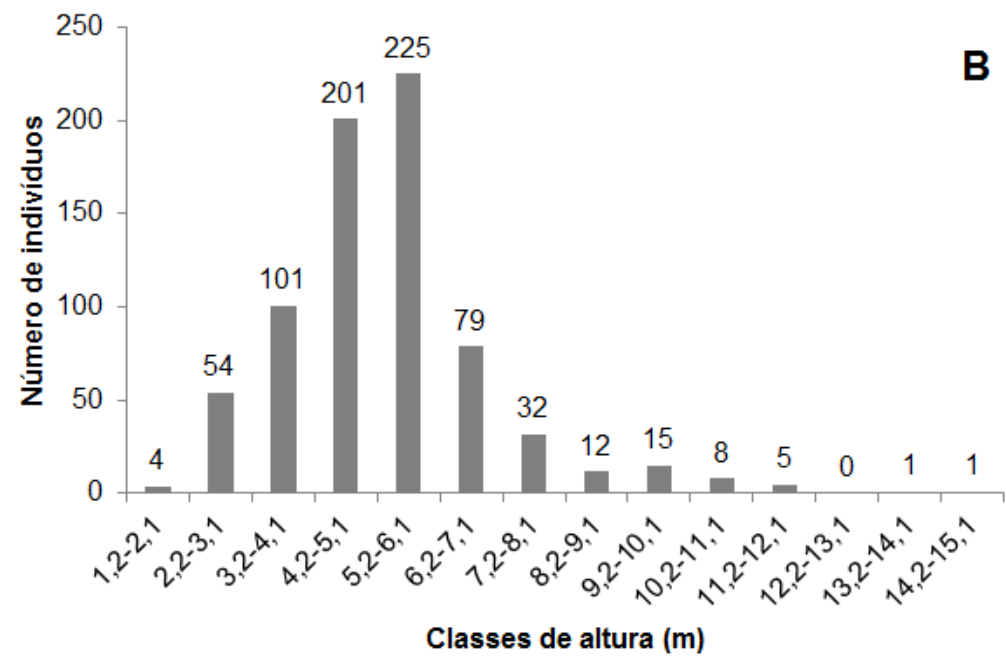

Tabela 4. Parâmetros florísticos e estruturais dos bairros amostrados na cidade de Itapuranga-GO

Table 4. Floristic and structural parameters of the sectors sampled in the Itapuranga city, Goias State

\begin{tabular}{lcccc}
\hline Parâmetros & $\begin{array}{c}\text { São Sebastião } \\
\text { do Xixá } \\
(\mathbf{1 9 7 0 )}\end{array}$ & $\begin{array}{c}\text { Parque } \\
\text { Alvorada } \\
(\mathbf{1 9 8 0 )}\end{array}$ & $\begin{array}{c}\text { Setor } \\
\text { Comercial } \\
(\mathbf{1 9 9 0 )}\end{array}$ & $\begin{array}{c}\text { Jardim Conde } \\
\text { dos Arcos } \\
(\mathbf{2 0 0 0})\end{array}$ \\
\hline Indivíduos & 223 & 261 & 160 & 94 \\
Famílias & 15 & 18 & 14 & 16 \\
Gêneros & 27 & 27 & 22 & 23 \\
Espécies & 28 & 28 & 24 & 25 \\
Índice de diversidade & 2,16 & 1,82 & 1,88 & 2,19 \\
$\left(\mathrm{H}^{\prime}\right)$ & & & & \\
\hline
\end{tabular}


A similaridade entre os bairros mostrou que o setor Jardim Conde dos Arcos foi o mais distinto em comparação aos demais (Figura 3). No entanto, os setores São Sebastião do Xixá e Parque Alvorada foram altamente similares com índices acima de 0,6 (Figura 3). Com base na análise, percebe-se que os bairros mais antigos, implantados nas décadas de 1970 e 1980, foram mais similares entre si (Figura 3), fato relacionado ao número de indivíduos e riqueza semelhantes. No entanto, recomenda-se que a arborização do setor Parque Alvorada seja manejada com o intuito de aumentar a biodiversidade local, a mais baixa calculada (Tabela 4 ).

Figura 3. Dendrograma baseado no índice de similaridade de Sørensen dos quatro bairros amostrados na cidade de Itapuranga-GO. Onde: Conde dos Arcos=Setor Jardim Conde dos Arcos; Sebastião=Setor São Sebastião do Xixá; Parque Alvorada $=$ Setor Parque Alvorada e Comercial=Setor Comercial

Figure 3. Dendrogram based on the Sørensen similarity of the four sectors sampled in the Itapuranga city, Goias State

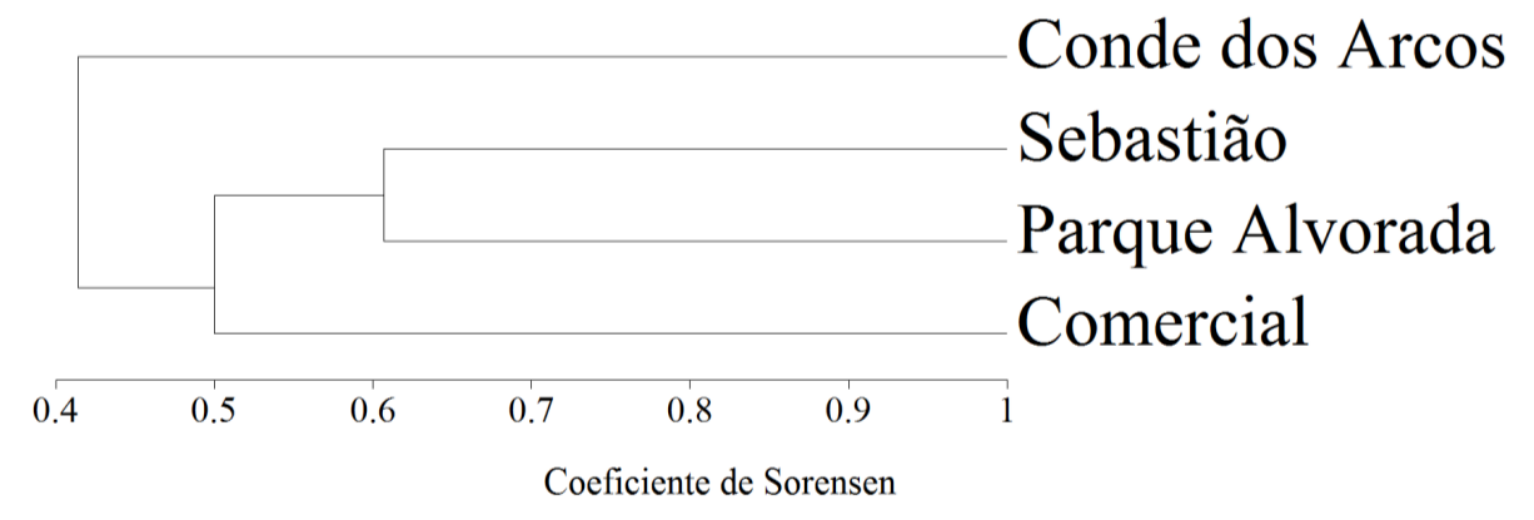

O setor Jardim Conde dos Arcos, bairro mais recente criado na década 2000, apresentou-se distinto dos demais (Figura 3). Este bairro exibiu o maior índice de diversidade calculado e a menor densidade de indivíduos lenhosos. Portanto, recomenda-se o plantio de mais indivíduos nas vias públicas do bairro, levando-se em consideração uma seleção adequada das espécies e a diversidade de espécies.

\section{CONCLUSÕES}

A arborização urbana implantada nas vias públicas da cidade de Itapuranga mostra a predominância de apenas duas espécies, uma exótica - Syzygium malaccense (L.) Merr. \& L.M. Perry e uma nativa da vegetação brasileira - Licania tomentosa (Benth.) Fritsch. A 
comparação entre bairros, com diferentes décadas de criação, mostrou heterogeneidade florística, e revelou aspectos importantes com relação à florística que devem ser levados em consideração para manejo mais adequado da arborização nos bairros.

\section{AGRADECIMENTOS}

Ao professor Manoel Cláudio da Silva Júnior da Universidade de Brasília pela identificação de material botânico e Plauto Simão de Carvalho da UEG-Unidade Universitária de Palmeiras de Goiás pelas valiosas sugestões durante a realização deste trabalho.

\section{REFERÊNCIAS}

ANGIOSPERM PHYLOGENY GROUP (APG). An update of the Angiosperm Phylogeny Group classification for the orders and families of flowering plants: APG III. Botanical Journal of the Linnean Society, v. 161, p. 105-121, 2009.

ÁRVORES DAS RUAS E PRAÇAS DE POÇOS DE CALDAS, com chave de identificação. Poços de Caldas: CEPA/ALCOA, 1999, 68p.

BATISTEL, L. M; DIAS, M. A. B; MARTINS, A. S; RESENDE, I. L. de M. Diagnóstico qualitativo e quantitativo da arborização urbana nos bairros Promissão e Pedro Cardoso, Quirinópolis, Goiás. Revista Brasileira de Arborização Urbana, Piracicaba, SP: v.4, n.3, p. 110-129, 2009.

COMPANHIA ENERGÉTICA DE MINAS GERAIS - CEMIG. Manual de arborização. Belo Horizonte: Fundação Biodiversitas, 2011. 112 p.

COLETTO, E. P; MULLER, N. G; WOLSKI, S. S. Diagnóstico da arborização das vias públicas do município de Sete de Setembro-RS. Revista Brasileira de Arborização Urbana, Piracicaba, SP: v. 3, n. 2, p. 110-122, 2008.

COSTA, P. M. A convenção climática e o surgimento de commodities ambientais. [S.I.], Gazeta Mercantil, 1997. 4 p.

COSTA E LIMA, R. M. Avaliação da Arborização Urbana do Plano Piloto. Dissertação de Mestrado em Ciências Florestais, Departamento de Engenharia Florestal, Faculdade de Tecnologia, Universidade de Brasília, Brasília, DF. 2009. 95 p. 
COSTA E LIMA R. M; SILVA JÚNIOR, M.C. Inventário da arborização urbana implantada na década de 60 no Plano Piloto, Brasília, DF. Revista Brasileira de Arborização Urbana, Piracicaba, SP: v.5, n.4, p.110-127, 2010.

DANTAS I. C.; SOUZA, C. M. C. Arborização urbana na cidade de Campina Grande-PB: Inventário e suas espécies. Revista de Biologia e Ciências da Terra, v.4, n.2, 2004.

FELFILI, J. M.; SILVA JÚNIOR, M. C. Diversidade alfa e beta no cerrado sensu stricto, Distrito Federal, Goiás, Minas Gerais e Bahia. In: SCARIOT, A.; SOUSA-SILVA, J. C.; FELFILI, J. M. Cerrado: Ecologia, Biodiversidade e Conservação. Brasília: Ministério do Meio Ambiente, 2005. p. 143-154.

CENTRAIS ELÉTRICAS DE SÃO PAULO -CESP. Guia de Arborização. 3.ed. São Paulo:, 1988. 33p. (Coleção Ecossistemas Terrestres, 006).

INSTITUTO BRASILEIRO DE GEOGRAFIA E ESTATÍSTICA - IBGE. Cidades @2010. Disponível em: <http: //http://www.ibge.gov.br/cidadesat/topwindow.htm?1> Acesso em: 25 de out. 2011.

INSTITUTO NACIONAL DE METEOROLOGIA - INMET. Disponível em: <http://www.inmet.gov.br/portal/> Acesso em 28 de set.2012.

ISERNHAGEN, I; LE BOURLEGAT, J. M. G; CARBONI, M. Trazendo a riqueza arbórea regional para dentro das cidades: possibilidades, limitações e benefícios. Revista Brasileira de Arborização Urbana. Piracicaba, SP: v.4, n.2, p.117-138, 2009.

KOVACH, W. L. MVSP - Multivariate Statistical Package, version 3.1. Kovach Computing Services, Pentraeth, 1993.

LORENZI, H. Árvores Brasileiras: Manual de identificação e cultivo de plantas arbóreas nativas do Brasil. 5 ed. São Paulo: Instituto Plantarum, 2008.

MAGURRAN, A.E.; MCGILL, B.J. Biological Diversity: frontiers in measurement and assessment. Oxford University Press, 2011. 345p.

MATOS, E; QUEIROZ, L. P. DE. Árvores para cidades. Salvador: Solisluna, 2009.

MAZZAROTTO, A. S.; CUBAS, S.; MARANHO, L. T. Florestas urbanas: método de avaliação para gestão das áreas verdes. Revista Floresta, v. 41, n. 3, p. 501-518, 2011. 
MUNEROLI, C. C; MASCARÓ, J. J. Arborização urbana: uso de espécies arbóreas nativas na captura do carbono atmosférico. Revista Brasileira de Arborização Urbana, Piracicaba, SP: v. 5, n. 1, p. 160-182, 2010.

NUNES, R. V; SILVA JÚNIOR, M. C; FELFILI, J. M; WALTER AND B. M. T. Intervalos de classe para a abundância, dominância e frequência do componente lenhoso do cerrado sentido restrito no Distrito Federal. Revista Árvore, v. 26, n. 2, p. 173-182. 2002.

PAIVA, H.N. Seleção de espécies para arborização urbana. Revista Ação Ambiental, Viçosa, MG: v.2, n.9, p.14-16, 1999/2000.

PIVETTA, K. F. L.; SILVA FILHO, D. F. Arborização urbana. Boletim Acadêmico: série arborização urbana, Jaboticabal-SP, 2002, 69 p.

RIBEIRO, J. F.; WALTER, B.M.T. As principais fitofisionomias do bioma Cerrado. In: SANO. S. M.; ALMEIDA, S. P.; RIBEIRO, J.F. (Eds.). Cerrado: ecologia e flora. Embrapa Cerrados, Brasília-DF, 2008. p. 153-212.

SANTOS, N.R.Z. dos; TEIXEIRA, I.F. Avaliação qualitativa da arborização da cidade de Bento Gonçalves, RS. Ciência Florestal, v.1., n.1., p.88-99, 1991.

SÃO PAULO. Prefeitura da cidade de São Paulo. Manual técnico de arborização urbana. 2 ed. 2005, 48p.

SERPA, D. S; MORAIS, N. A; MOURA, T. M. Arborização urbana em três municípios do Sul do estado de Goiás: Morrinhos, Goiatuba e Caldas. Revista Brasileira de Arborização Urbana, Piracicaba, SP: v. 4, n. 3, p. 98-112, 2009.

SILVA, L. M.; HASSE, I.; MOCCELLIN, R.; ZBORALSKI, A. R. Arborização de vias públicas e a utilização de espécies exóticas: o caso do bairro centro de Pato Branco/PR. Scientia Agraria (UFPR), v. 8, p. 47-53, 2007.

SILVA JÚNIOR, M. C. da. 100 árvores do Cerrado: guia de campo. 2 ed. Brasília: Rede de sementes do Cerrado, 2012.

SILVA JÚNIOR, M. C. da; COSTA E LIMA, R. M. 100 Árvores Urbanas Brasília: Guia de campo. Brasília, DF: Rede de Sementes do Cerrado, 2010.

SILVA FILHO, D.F. da S; PIZETTA, P.U.C.; ALMEIDA, J.B.S.A. de. PIVETTA, K.F.L; FERRAUDO, S. Banco de dados relacional para cadastro, avaliação e manejo da arborização em vias públicas. Revista Árvore, v. 26, n. 5, p. 629-642, 2002. 
SILVA FILHO, D. F.; BORTOLETO, S. Uso de indicadores de diversidade na definição de plano de manejo da arborização viária de Águas de São Pedro - SP. Revista Árvore, v. 29, n. 6, p. 973-982, 2005.

SOUZA, A. R. C.; ROBAINA, A. D.; PEITER, M. X.; FERRAZ, R. C.; SCHWAB, N. T.; SOUZA, G. R. C.; PINTO, L. M. Identificação das espécies ornamentais nocivas na arborização urbana de Santiago/RS. Revista Brasileira de Arborização Urbana, Piracicaba, SP: v. 6, n. 2, p. 44-56, 2011.

SOUZA, V. C; LORENZI. H. Botânica sistemática: guia ilustrado para ilustração das famílias de fanerógamas nativas e exóticas no Brasil, baseado em APG II. 2. ed. Nova Odessa, SP: Instituto Plantarum, 2008. p. 381-485. 